\title{
Failure Analysis of Trace Elements and Surfaces Using Hitachi Dedicated STEM
}

C. Vartuli ${ }^{1 *}$ and B. Tracy ${ }^{2}$ and Jitty $\mathrm{Gu}^{2}$

${ }^{1 .}$ Hardware Division, FA Group, Apple, Cupertino, CA, USA.

2. Advanced Imaging Group, EAG Laboratories, Sunnyvale, Ca and Raleigh, NC, USA.

* Corresponding author: cvartuli@apple.com

Failure analysis is often a time limited activity, and as feature size decreases, surface effects and trace contaminants have larger impacts. Understanding sample topography/projection effects as well as determining any elemental anomalies can go a long way to identify the cause of failure so the process line can be corrected. Traditional analysis for crystallographic and elemental characterization such as XRD and SIMS may be severely limited or unavailable given the dimensions of the features.

While the characterization of surfaces by a variety of imaging techniques is a broad and well-established field, recent reports of angstrom-level resolutions [1] have opened this discipline to novel studies of surface structure. This is full factor-of-four improvement in resolution as compared to a conventional UHR SEM. This improvement has accomplished thru the addition of a secondary electron detector to a probe-corrected Scanning Transmission Electron Microscope (STEM). This work has been expanded to include detailed surface studies as well as atomic-level chemistry measurements [2,3]

The STEM/SEM is can be operated in at least three imaging modes. In Figure 1a. we present an intermediate magnification HVSEM image showing a cluster of silicon nano particles. Despite the acceleration voltage of $200 \mathrm{kV}$, the image looks just as you might expect a topography-rich secondary electron image to appear. Figure $1 \mathrm{~b}$. shows a Z-contrast image where the bright portions result from particle overlap and hence increase scattering due to increased thickness. Figure 1c. shows bright field STEM. Please note the twinned silicon region. Zooming up in magnification, the familiar silicon dumbbell structure is revealed in the HAADF image (Fig 1d). Switching to secondary electron detection from the same area, Figure 1e. shows the silicon dumbells right at the noise/resolution limit. The HFOV for both Figures 4 and 5 is $16 \mathrm{~nm}$

Determining the root cause of corrosion or contaminant defects quickly and accurately is routinely done by Energy Dispersive X-ray (EDX) in SEM or STEM. In some cases, the concentration of the element causing the failure may be very low. In the case of corrosion caused by F, for example, the mobility of the $\mathrm{F}$ ions under the beam during preparation and examination can quickly reduce the concentration below detectable levels. In Figure 2a the STEM image of a corroded via is shown with the corresponding elemental analysis. Only trace F contamination remains due to the mobility of the ions, and special care is needed to optimize preparation and analysis of the site to preserve the evidence.

\section{References:}

[1] XF Zhang, Microscopy Today 19 (2011), p. 26.

[2] J Ciston et al., Nature Communications 6 (2015), p. 1.

[3] J Howe et al., Microsc. Microanal. 21 (S3) (2015), p. 7358. 


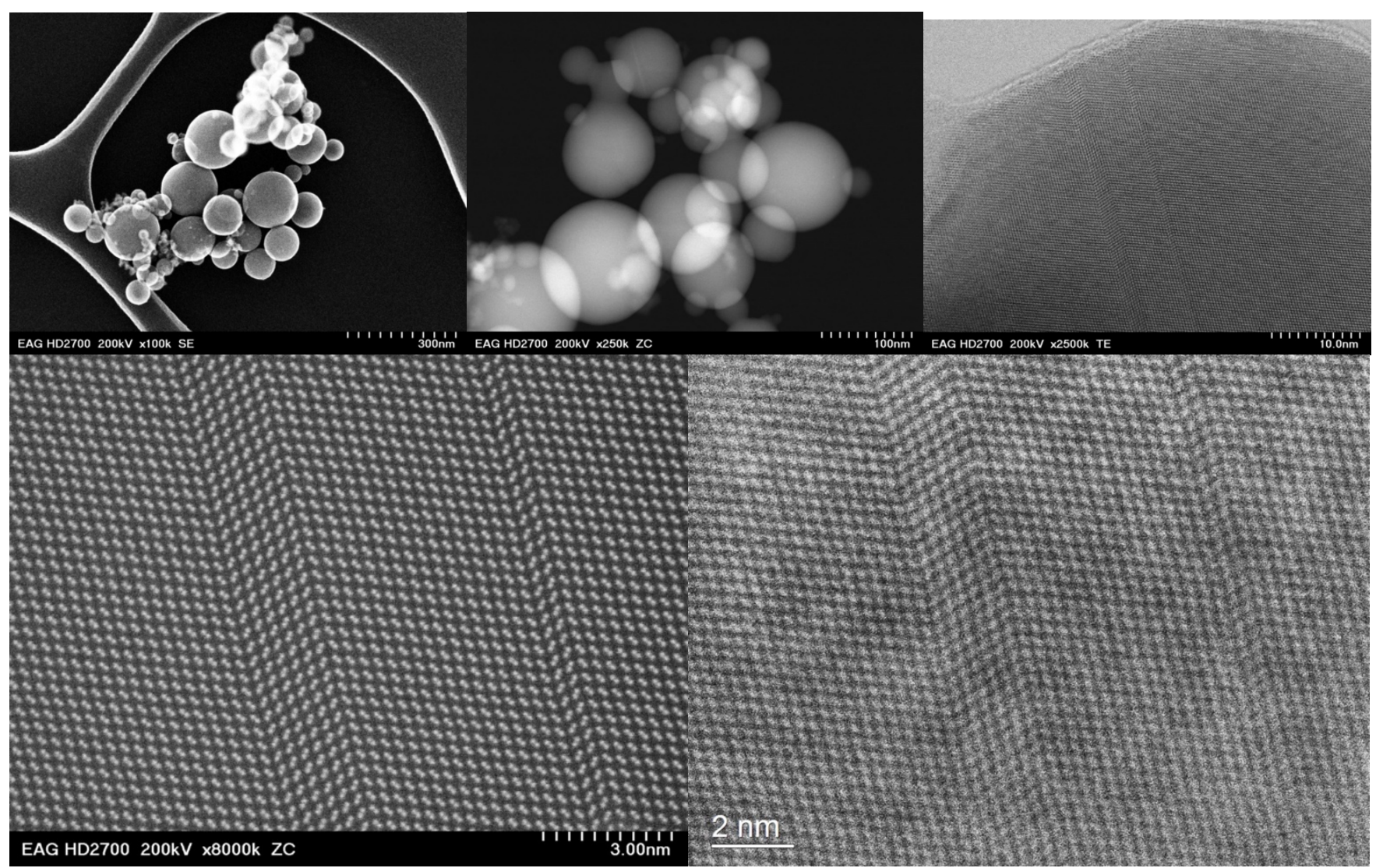

Figure 1. An intermediate magnification HV SEM image showing a cluster of silicon nano particles with a HFOV of 1 micron. 1b. Z- contrast image where the bright portions result from particle overlap and hence increase scattering due to increased thickness. 1c. shows bright field STEM image in a very thin portion of the sample. 1d. Zooming up in magnification, the familiar silicon dumbbell structure is revealed in the HAADF image. 1e. Switching to secondary electron detection from the same area, Figure 5 shows the silicon dumbells right at the noise/resolution limit.
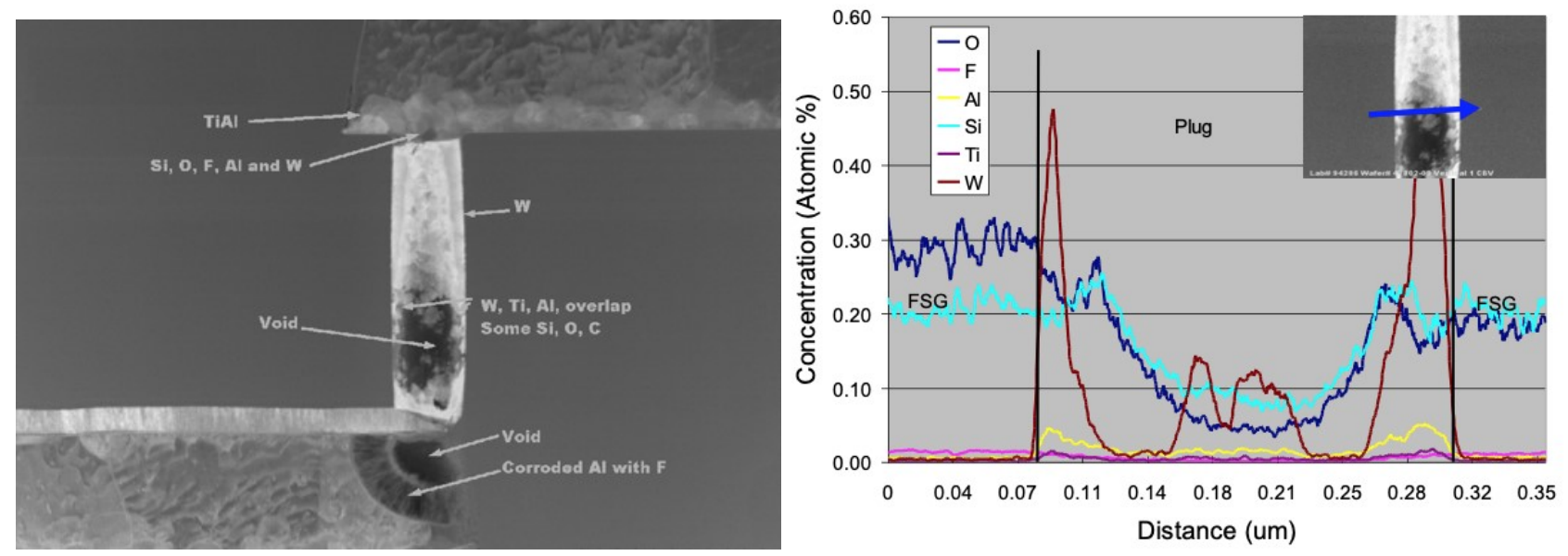

Figure 2. Corroded via and $2 \mathrm{~b}$. corresponding elemental analysis to identify the root cause. 